\title{
Rapamycin inhibits cell proliferation in type I and type II endometrial carcinomas: A search for biomarkers of sensitivity to treatment
}

\author{
Victoria L. Bae-Jump ${ }^{a, b}$, Chunxiao Zhou ${ }^{a, c, 1}$, John F. Boggess ${ }^{a, c}$, Young E. Whang ${ }^{b, c}$, Lisa \\ Barroilhet $^{d}$, and Paola A. Gehrig ${ }^{a, c},{ }^{*}$ \\ aDepartment of Obstetrics and Gynecology, Division of Gynecologic Oncology, University of \\ North Carolina at Chapel Hill, Chapel Hill, NC, USA \\ ${ }^{b}$ Department of Medicine, Division of Hematology Oncology, University of North Carolina at \\ Chapel Hill, Chapel Hill, NC, USA \\ 'Lineberger Comprehensive Cancer Center, University of North Carolina, Chapel Hill, NC, USA \\ dDivision of Gynecologic Oncology, Brigham and Women's Hospital, Boston, MA, USA
}

\begin{abstract}
Objectives-Our goal was to evaluate the effect of rapamycin, an mTOR inhibitor, in type I and II human endometrial cancer tumor explants.

Methods-Short-term tissue culture with fresh endometrial cancer tumor explants was performed. Cell proliferation was assessed by MTS assay after treatment with rapamycin. Akt and PTEN status were documented by Western blotting. The effect of rapamycin on phosphorylatedS6 and 4E-BP-1 was also assessed by Western blotting. Real-time RT-PCR was used to quantify hTERT mRNA expression. Telomere length was determined by terminal restriction fragment Southern blotting.
\end{abstract}

Results-Thirteen fresh endometrial cancer tumor explants (nine Type I, four Type II) were placed in short-term culture and treated with rapamycin. Nine of the endometrial cancer tumors responded to rapamycin, with a median $\mathrm{IC}_{50}$ of $11.4 \mathrm{nM}$. Sensitivity to rapamycin was independent of PTEN and Akt status. Tumors (13/13) had a reduction in phosphorylated-S6 and 10/13 had a reduction in phosphorylated 4E-BP-1. Rapamycin decreased hTERT mRNA expression in all of the endometrial cancer tumors. Telomere length did not correspond with responsiveness to this drug.

Conclusions-Rapamycin demonstrated activity in fresh endometrial tumor explants independent of PTEN and Akt status. Some tumors demonstrated a reduction in phosphorylated-

\footnotetext{
This work was presented in part at the 2005 Annual Meeting of the Society of Gynecologic Oncologists, Miami Beach, FL. (c) 2010 Elsevier Inc. All rights reserved.

* Corresponding author. University of North Carolina at Chapel Hill, Department of Obstetrics and Gynecology, Division of Gynecologic Oncology, CB \#7572, Chapel Hill, NC 27599-7572, USA. Fax: +1 919966 2646. pam68@ med.unc.edu. .

1 This author contributed an equal amount of work as first author.

Conflict of interest statement We, the authors of this manuscript, have no financial or personal relationships to disclose that could inappropriately influence or bias this work.
} 
S6 and 4E-BP-1 without a significant change in cellular proliferation, suggesting that additional pathways may modulate cellular proliferation. Thus, mTOR inhibitors may be a useful targeted therapy for both type I and type II endometrial cancers, but the search remains for a predictive biomarker of sensitivity to this therapy.

\section{Keywords}

Rapamycin; PTEN; Akt; hTERT; Endometrial carcinoma; mTOR pathway

\section{Background}

Endometrial carcinoma is the most common gynecologic malignancy in the United States, accounting for more than 42,160 cases and 7780 deaths in 2009 [1]. Women with early stage disease (FIGO stage I and II) and endometrioid histology (type I) have a relatively good prognosis with surgery alone or surgery plus radiation. However, those patients with advanced or recurrent disease are unlikely to be cured by surgery, conventional chemotherapy, radiation or a combination of these modalities. In addition, $20 \%$ of women with be diagnosed with a tumor of either serous or clear cell histology (type II) and even in the setting of early stage disease (FIGO stage I), these women have a 5-year survival rate of $60 \%$ as compared with an $85-90 \%$ survival rate in those with stage I endometrioid adenocarcinoma. Unlike type I endometrial cancers that are associated with obesity and estrogen excess, there is no clear epidemiologic profile for type II cancers which behave much more aggressively. As some estimate that the percent of women dying from endometrial carcinoma has increased by $227 \%$ over the past decade, there is a great need to develop novel targeted agents that can be used alone or in combination with current treatment strategies.

A dualistic model for the development and progression of endometrial cancer exists that divides these tumors into type I and type II based on biological, molecular and clinical parameters [2]. Type I or those tumors of endometrioid histology comprise $80 \%$ of cases and are thought to arise from persistent unopposed estrogen stimulation, which can be either endogenous or exogenous. Women who develop these tumors are typically perimenopausal and often have risk factors such as obesity, hyperlipidemia, nulliparity, diabetes mellitus and insulin resistance, polycystic ovarian syndrome, hypertension and late-onset menopause. These tumors are preceded by an identifiable premalignant state of hyperplasia and ultimately, atypical hyperplasia, and are generally estrogen receptor (ER) and progesterone receptor (PR) positive. Genetic alterations associated with these tumors include PTEN deletions or mutations (36-83\%), microsatellite instability (20-40\%), mutations of K-ras (15-30\%) and gain of function mutations in $\beta$-catenin (25-40\%) [3-5].

In contrast, type II or those tumors of atypical histologies such as serous and clear cell carcinomas have no association with excess endogenous or exogenous estrogen and have no recognized risk factors. These tumors generally occur in postmenopausal women in the setting of an atrophic endometrium. Type II tumors behave much more aggressively and show a propensity for deep invasion, lymphovascular permeation and distant spread. ER and PR are generally negative or weakly positive. A precursor lesion is not as well defined but an in situ form of this type of carcinoma has been described and is referred to as endometrial 
intraepithelial carcinoma (EIC) [6]. The molecular genetic profile for these tumors is different than type I tumors and is associated with aneuploidy, p53 mutations (80-90\%), p16 inactivation (40\%), overexpression of HER-2/neu (40-80\%) and E-cadherin alterations (80$90 \%)[3-5]$.

As our knowledge has evolved of the underlying molecular mechanisms involved in endometrial cancer development and progression, many potential targeted therapeutic agents are being actively investigated [7]. Loss of PTEN expression is one of the most prevalent molecular abnormalities associated with endometrial cancers and occurs in an estimated 36$83 \%$ of type I endometrial cancers. Unlike most other tumor types, loss of PTEN expression is observed in premalignant lesions of the endometrium, suggesting that PTEN loss may be a potential initiator of endometrial cancer development [8]. Wild-type PTEN downregulates the PI3K/Akt/mTOR signaling pathway, which transduces extracellular growth regulatory signals to intracellular mediators of growth and cell survival [9]. Loss of PTEN results in constitutive activation of Akt and subsequently leads to promotion of cellular proliferation and resistance to apoptosis. Therefore, mTOR is a promising target for endometrial cancer therapy, and clinical trials of mTOR inhibitors are currently underway for this disease.

mTOR inhibitors such as rapamycin exert their anti-proliferative effects through inhibition of the serine/threonine kinase, mammalian target of rapamycin (mTOR), by forming a complex with one of the immunophilin family of FK506 binding proteins, FKBP12 [10,11]. The inhibition of mTOR prevents phosphorylation and activation of S6K1 and 4E-binding protein 1 (4E-BP-1), which ultimately results in the inhibition of translation of critical mRNAs involved in cell cycle progression [10,11]. We have previously demonstrated that rapamycin profoundly inhibits cell proliferation through G1 cell cycle arrest in established endometrial cancer cell lines [12]. Our goal was to expand on our previous work and assess the effect of rapamycin in human primary cultures of both type I and type II endometrial cancer tumors.

\section{Material and methods}

\section{Drug and reagents}

Rapamycin was purchased from Sigma (St. Louis, MO) and dissolved in DMSO. The antiwild-type PTEN antibody, anti-phosphorylated-ser473-Akt antibody, anti-phosphorylatedser235/236-S6 antibody, anti-total-S6 antibody, anti-phosphorylated-ser65-4E-BP-1 antibody and anti-total-4E-BP-1 antibody were purchased from Cell Signaling (Danvers, MA). The anti-estrogen receptor antibody, the anti-progesterone receptor antibody and the anti- $\beta$-actin antibody were from Santa Cruz Biotechnology (Santa Cruz, CA). Enhanced Chemiluminescence Western blotting detection reagents (ECL) were from Amersham (Arlington Heights, IL).

\section{Sources of tissue}

Thirteen tumor specimens were sampled from patients undergoing surgery for endometrial carcinoma at the University of North Carolina at Chapel Hill. The protocol was reviewed and exemption granted by the Institutional Review Board at the University of North 
Carolina at Chapel Hill. The international Federation of Gynecology and Obstetrics (FIGO) stage and histological grade of the tumors are shown in Table 1.

\section{Culture of primary endometrial tumor cells}

In brief, the freshly obtained tissues were washed three times in sterile, cold Hank's Buffered Salt Solution (HBSS) to remove blood and secretions, and then gently minced by scissors in M199 medium containing 10\% fetal calf serum (FCS) and $0.3 \mathrm{~g} / \mathrm{l}$ glutamine. These tumor cells were then incubated for $2-4 \mathrm{~h}$ at $37{ }^{\circ} \mathrm{C}$ in M199 supplemented with $0.1 \%$ collagenase IA, $100 \mathrm{U} / \mathrm{ml}$ penicillin and streptomycin with occasional shaking. After two centrifugations with PBS wash, the tumor cells were suspended in M199 medium and then diluted to $1 \times 10^{5}$ cells $/ \mathrm{ml}$. Aliquots $(100 \mu \mathrm{l})$ of tumor cell suspension were plated into 96 well tissue culture plates resulting in approximately $1 \times 10^{4}$ cells per well. The cells were incubated at $37{ }^{\circ} \mathrm{C}$ for $24 \mathrm{~h}$ in a $5 \% \mathrm{CO}_{2}$ incubator, and then were treated with various concentrations of rapamycin or DMSO. Cell proliferation was assayed by the CellTiter 96 AQ non-Radioactive cell proliferation assay (MTS) from Promega (Madison, WI). After 24, 48 and $72 \mathrm{~h}, 20 \mu \mathrm{l}$ of freshly prepared MTS/PMS solution was added to each well, and the mixture was incubated for $2 \mathrm{~h}$ at $37^{\circ} \mathrm{C}$. Optical density was read directly at $490 \mathrm{~nm}$ using ELISA plate reader. All samples were assayed at least in quadruplicate with an appropriate blank. The tumor inhibition rate (IR \%) was calculated for each assay by the formula:

$$
\text { IR. }(\%)=(1-T / C) \times 100 \%
$$

where $T=\mathrm{OD} 490$ of the treated cells and $C=\mathrm{OD} 490$ of the control cells. The tumor was judged to be sensitive when the IR was $250 \%$ and insensitive when the IR was $<50 \%$ at any of the time points of 24,48 or $72 \mathrm{~h}$.

\section{Western blotting analysis}

Tumor cells at a density of $3 \times 10^{5}$ cells/well in 6-well plates were cultured for $24 \mathrm{~h}$ and then treated with $20 \mathrm{nM}$ of rapamycin or vehicle (DMSO). Cell lysates were prepared in RIPA buffer ( $1 \% \mathrm{NP}-40,50 \mathrm{mmol} / \mathrm{l}$ Tris, $150 \mathrm{mmol} / \mathrm{l} \mathrm{NaCl})$. Equal amount proteins were separated by gel electrophoresis and transferred onto a nitrocellulose membrane. The membrane was blocked with 5\% nonfat milk and then incubated with 1:1000 dilution of primary antibody overnight at $4{ }^{\circ} \mathrm{C}$. Subsequently, the membrane was washed and incubated with a secondary peroxidase-conjugated antibody for $1 \mathrm{~h}$ after washing. Antibody binding was detected by using an enhanced chemiluminescence detection system. Western immunoblotting films were digitized, and band net intensities were quantified suing a Millipore Digital Bioimaging System (Bedford, MA). After developing, the membrane was stripped and re-probed using $\beta$-actin to confirm equal loading. Western blot films were digitized, and band net intensities were quantified by a densitometer using the Genegynome Image System (Sygene, MD). A target protein $/ \beta$-actin ratio was calculated for each protein. An endometrial tumor was considered positive for a particular protein if the target protein $/ \beta$ actin ratio was $\searrow 0.020$. 


\section{Telomere length}

Telomere length was assessed by using the TeloTAGGG Telomere Length Assay kit (Roche Applied Science, Indianapolis, IN), according to the manufacturer's protocol. DNA was extracted from the human endometrial cancer tumor specimens using the standard method of phenol-chloroform. $2 \mu \mathrm{g}$ of DNA was digested by mixing HinfI (20 U) and RsaI (20 U) and incubated at $37{ }^{\circ} \mathrm{C}$ for $2 \mathrm{~h}$. Resulting DNA fragments were then separated on a $0.8 \%$ agarose gel by electrophoresis in $1 \times$ TAE running buffer. Separated DNA was subsequently transferred to a positively charged nylon membrane and blotted with digoxigenin-labeled (CCCTAA) 3 DNA probe for $3 \mathrm{~h}$ at $62{ }^{\circ} \mathrm{C}$. The membrane was exposed to a phosphorimager screen and detected on the PhosphorImager Storm 860 (Molecular Dynamics, Sunnyvale, $\mathrm{CA}$ ) and then on an X-ray film. Median telomere length was calculated using the Image $\mathrm{J}$ freeware (National Institutes of Health, Bethesda, MD).

\section{Real-time PCR for hTERT}

Total RNA was isolated using an RNA mini kit, and further DNase treatment was performed using the RNase-Free DNase Set (QIAGEN, Chatsworth, CA) following the manufacturer's instructions. The reverse transcription and PCR reactions were conducted using the TaqMan Gold one-step PCR kit in the ABI 7700 Sequence Detection System (Applied Biosystems, Foster City, CA). Reverse transcription was carried out at $48{ }^{\circ} \mathrm{C}$ for $30 \mathrm{~min}$. The PCR condition consisted of a $10 \mathrm{~min}$ step at $95^{\circ} \mathrm{C}$ and 40 cycles at $95{ }^{\circ} \mathrm{C}$ for $15 \mathrm{~s}$ and $65^{\circ} \mathrm{C}$ for 1 min in a final volume of $30 \mu \mathrm{l}$ containing 50-100 ng total RNA, $3 \mu \mathrm{l} 10 \times$ TaqMan buffer A, $5.5 \mathrm{mM} \mathrm{MgCl}_{2}, 300 \mu \mathrm{M}$ dATP, dCTP and dGTP, $600 \mu \mathrm{M}$ dUTP, $100 \mathrm{nM}$ probe, $200 \mathrm{nM}$ forward and reverse primer, 15 units RNase inhibitor, 7.5 units MuLV reverse transcriptase and 1 unit AmpliTaq Gold DNA Polymerase. A housekeeping gene known as acidic ribosomal phosphoprotein PO (RPLPO) was used as an active and endogenous reference to correct for differences in the amount of total RNA added to each reaction and to compensate for different levels of inhibition during reverse transcription of RNA into cDNA during PCR. Primers and fluorogenic probes for hTERT and RPLPO have been described previously [13]. The standard curve for quantification of hTERT was generated by using serial dilutions of a known amount of hTERT cRNA synthesized by in vitro transcription of a cloned fragment. The level of the hTERT expression of each sample was calculated as a ratio that the amount of hTERT transcript was divided by the amount of the endogenous housekeeping gene RPLPO.

\section{Statistical analysis}

Results for experiments were normalized to the mean of the control and analyzed using the Student's $t$-test. Differences were considered significant if the $p$ value was less than 0.05 $(\mathrm{p}<0.05)$ with a confidence interval of $95 \%$. STATA software (StataCorp, College Station, TX) was used to perform the statistical analyses. 


\section{Results}

\section{Characterization of the Type I and Type II endometrial cancer tumor explants}

Thirteen endometrial cancer explants were harvested at the time of hysterectomy. Nine samples were type I endometrioid adenocarcinomas and four samples were type II serous or clear cell adenocarcinomas (Tables 1 and 2). ER, PR, wild-type PTEN and phosphorylatedAkt status was determined for each sample by Western immunoblotting (Fig. 1). All of the type I and type II tumors expressed PR (target protein/ $\beta$-actin ratio range of 0.976-0.081), and $6 / 9$ of the type I and $3 / 4$ of the type II tumors expressed ER (target protein/ $\beta$-actin ratio range of 0.00-0.893). Five of the nine (55\%) type I tumors expressed wild-type PTEN while the other four type I tumors (44\%) were PTEN negative (target protein/ $\beta$-actin ratio range of $0.00-0.728)$. Of the grade 1 endometrioid lesions, two of the three $(67 \%)$ were PTEN negative as compared to $3 / 6(50 \%)$ of the grade 2 or grade 3 lesions. Two of the four type II tumors (50\%) were PTEN negative. Lastly, 8/9 of the type I tumors (89\%) and 4/4 (100\%) of the type II tumors expressed phosphorylated-Akt (target protein/ $\beta$-actin ratio range of $0.00-0.794)$.

\section{Anti-proliferative effect of rapamycin}

We examined the effect of rapamycin in the type I and type II endometrial cancer tumor explants at 24, 48 and $72 \mathrm{~h}$ (Table 1). The $\mathrm{IC}_{50}$ at $48 \mathrm{~h}$ is reported in Table 1. Of these tumors, $9 / 13$ responded to rapamycin, with a median $\mathrm{IC}_{50}$ of $11.4 \mathrm{nM}$ (range $0.01-50 \mathrm{nM}$ ). For the type I tumors, $7 / 9$ (78\%) responded to rapamycin (median $\mathrm{IC}_{50}$ of $12.5 \mathrm{nM}$ ), and for the type II tumors, $2 / 4(50 \%)$ responded to treatment (median $\mathrm{IC}_{50}$ of $7.5 \mathrm{nM}$ ). Sensitivity to rapamycin was independent of PTEN and phosphorylated-Akt status. Rapamycin inhibited cell growth in 5/7 of the PTEN positive (71\%) and 4/6 of the PTEN negative (67\%) endometrial cancers. Only one endometrial tumor was phosphorylated-Akt negative, and this tumor did not respond to rapamycin. Of the phosphorylated-Akt positive tumors, 9/12 $(75 \%)$ had a response to treatment with rapamycin.

\section{Effect of rapamycin on the mTOR pathway}

To investigate the mechanisms underlying the anti-proliferative effect of rapamycin, we characterized the effect of this combination treatment on relevant cell signaling targets. Previous studies suggest that p70S6K is a downstream target of the mTOR pathway [14]. p70S6K kinase directly phosphorylates the 40S ribosomal protein S6, which results in enhanced synthesis of proteins that contain a polypyrimidine tract in the $5^{\prime}$-untranslated region [14]. Therefore, we studied the effect of rapamycin on the phosphorylation of the S6 ribosomal protein among the type I and type II endometrial cancer tumor explants. After 24, 48 and $72 \mathrm{~h}$ of treatment, rapamycin dramatically decreased the phosphorylation of S6 in all the endometrial cancer tumor explants by 3.4-13.2 fold ( $p=0.0001$ ) (Fig. 2A). Little effect was seen on the expression of total-S6.

mTOR kinase regulates protein synthesis by phosphorylating the translation repressor 4EBP-1 on multiple serine/threonine sites [15]. The phosphorylation status of 4E-BP-1 regulates binding to eukaryotic initiation factor $4 \mathrm{E}$ (eIF4E). Hyperphosphorylation of 4EBP-1 disrupts its binding to eIF4E, activating cap-dependent protein synthesis [16]. After 
24, 48 and $72 \mathrm{~h}$ of treatment, rapamycin decreased phosphorylation of 4E-BP-1 (Fig. 2B) in $11 / 13$ of the endometrial cancer tumor explants by $2.8-6.6$ fold $(p=0.0012)$. Rapamycin had little effect on the expression of total-4E-BP-1. Thus, these results suggest that rapamycin's effect on either phosphorylated-S6 or phosphorylated-4E-BP-1 did not correlate with response to treatment.

\section{Effect of rapamycin on telomere length and hTERT mRNA expression}

The hTERT gene encodes the catalytic subunit of telomerase. hTERT expression is the ratelimiting determinant of the enzymatic activity of human telomerase and is thought to be a sensitive marker of telomerase function. Real-time RT-PCR was used to quantify hTERT mRNA expression in the endometrial cancer tumor explant. Treatment with rapamycin (20 $\mathrm{nM}$ ) decreased hTERT mRNA expression in all of the type I and type II endometrial cancer tumor explants (Fig. 3). Among these tumors, hTERT mRNA expression was decreased by a mean of $27 \%$ ( $p=0.0001)$ (Fig. 3).

Telomere length in the endometrial cancer tumor explants was compared by terminal restriction fragment southern blot analysis (Fig. 4). The mean telomere length was $7.2 \mathrm{~kb}$ for the type I tumors (range 4.9-10.5 kb) and $8.5 \mathrm{~kb}$ for the type II tumors (range 3.8-11.2 kb). In addition, the mean telomere length was 7.5 for the rapamycin sensitive tumors (3.8-10.8 $\mathrm{kb})$ and $7.8 \mathrm{~kb}$ for the rapamycin resistant tumors $(4.9-11.2 \mathrm{~kb})$. Thus, the effect of rapamycin on hTERT mRNA expression or baseline tumor telomere length did not predict responsiveness to rapamycin in these samples.

\section{Discussion}

We have demonstrated activity of rapamycin in both type I and type II endometrial cancer tumor explants (Tables 1 and 2). Rapamycin universally decreased phosphorylation of S6 and reduced hTERT mRNA expression in all of these tumors, but only inhibited cell proliferation in $69 \%$ of the tumors examined. We found no correlation between PTEN status or phosphorylated Akt status and response to treatment with rapamycin. Similarly, effects of rapamycin on phosphorylated S6, phosphorylated 4E-BP-1, hTERT mRNA expression or baseline telomere length were not predictive of sensitivity to rapamycin. To our knowledge, this is the first study to evaluate rapamycin in type I and II endometrial cancer tissue explants and assess potential biomarkers of response for this disease.

Hyperactivation of the PI3K/Akt/mTOR pathway is a feature of many types of cancer, including endometrial cancer. This can occur by a variety of mechanisms, including PTEN inactivation which has been found in up to $83 \%$ of type I endometrial cancers and $55 \%$ of precancerous lesions $[8,17]$. Alterations in PTEN occur most frequently by mutations that lead to loss of function. PTEN and PI3K act as opposing forces to regulate Akt phosphorylation. PI3K mutations have also been documented in approximately $36 \%$ of type I endometrial cancers and commonly occur in tumors that have PTEN mutations [18]. Loss of PTEN function in PTEN-null cancer cells has been proposed as an indicator of sensitivity to rapamycin and its analogues [19-23]. In contrast, others, including our previous work in endometrial, ovarian and cervical cancer cell lines, have found that loss of PTEN function was insufficient to predict response to mTOR inhibitors both in vitro and in vivo [12,24-26]. 
This present study further supports the lack of correlation between PTEN loss and response to rapamycin. Among the endometrial cancer tumors examined, a similar percentage of PTEN positive (71\%) and PTEN negative (67\%) tumors were sensitive to the growth inhibitory effects of rapamycin.

The basal level of phosphorylated Akt in cancer cells has also been implicated as another potential biomarker of responsiveness to mTOR inhibitors, such as rapamycin [23,27]. However, we did not find this to be true among the type I and II endometrial cancer tumor explants that were evaluated. The vast majority $(92 \%)$ of the endometrial cancer tumors expressed basal levels of phosphorylated Akt. Of the endometrial tumors that did not respond to rapamycin, $2 / 4$ were strongly positive (target/ $\beta$-actin ratio of 0.460 and 0.794 ), $1 / 4$ was weakly positive (target/ $\beta$-actin ratio of 0.077 ) and $1 / 4$ was negative for phosphorylated-Akt. It could be argued that a change in phosphorylation after treatment with rapamycin may be a better predictor of response to therapy. However, in a recent study that examined the rapamycin analogue, everolimus, Akt phosphorylation induced by everolimus did not correlate with sensitivity to this treatment in a large panel of different cancer cell lines [27].

Cell cycle progression necessitates a steady increase in the rate of protein synthesis, resulting in the coordinated activation of cyclins, cyclin-dependent kinases (CDK) and CDK inhibitors. Rapamycin is known to block cell cycle progression through G1 by inhibition of two crucial downstream targets, S6 and 4E-BP-1, which are both mediators of protein translation. In the search for biomarkers to predict sensitivity to mTOR inhibitors, phosphorylated S6 and 4E-BP-1 have also been investigated with controversial results. Rapamycin and its analogues have been shown to inhibit S6 phosphorylation in tumors, skin and peripheral blood lymphocytes in animal models, and this effect was correlated with the anti-tumor benefit of rapamycin in these models [28,29]. In addition, it has been proposed that inhibition of S6 kinase activity in peripheral blood lymphocytes may serve as a surrogate biomarker for planning optimal dosing regimens of mTOR inhibitors, and this is being explored in recent clinical trials of the rapamycin analogues, everolimus and temsirolimus (CCI-779) [29,30]. Inhibition of phosphorylation of 4E-BP-1 by temsirolimus has also been correlated with the degree of tumor growth inhibition in prostate, glioma and ovarian carcinoma xenograft animal models [31]. We found that rapamycin decreased phosphorylation of S6 in all of the endometrial cancer tumor explants examined and decreased phosphorylation of 4E-BP-1 in most of these tumors (85\% or 11/13 tumors). These results are most consistent with what has been shown in studies of breast, prostate and multiple myeloma cells, where S6 and 4E-BP-1 phosphorylation was inhibited in both rapamycin-sensitive and rapamycin-resistant cancer cell lines [20,22,23]. Thus, inhibition of phosphorylation of S6 and 4E-BP-1 may indicate that adequate target inhibition has been achieved, but may not serve as useful biomarkers of sensitivity to mTOR inhibitor therapy. These findings suggest that additional pathways other than the mTOR pathway may modulate cellular proliferation or alternatively, that S6 and 4E-BP-1 may not be the only critical downstream effectors of mTOR signaling. In further support of this hypothesis, in a phase I trial of everolimus in 55 patients with advanced solid tumors, treatment resulted in almost complete inhibition of phosphorylation of S6 and 4E-BP-1 in tumor and skin samples but this did not predict sensitivity to therapy [30,32]. 
Telomeres are structures that cap the ends of chromosomes. As cells get older, telomeres progressively shorten and ultimately reach a crisis point that leads an older potentially defective cell towards cell death. Telomerase is an enzyme that adds DNA sequence repeats (TTAGGG) to the $3^{\prime}$ end of the DNA telomere region, thereby preventing the shortening of the caps at the ends of chromosomes. In most normal somatic cell types, telomerase activity is usually undetectable; however, the endometrium is a unique exception [33]. Telomerase activity rises and falls throughout the menstrual cycle, with high levels found during the proliferative phase and low levels during the secretory phase. It is hypothesized that these fluctuations in telomerase activity may be linked to the ability of the normal endometrium to repeatedly proliferate from the onset of menarche to menopause. Activation of telomerase enables cells to divide indefinitely and has been implicated as a key element in cellular immortality and oncogenesis for many cancers [33], including endometrial cancers [34,35]. Expression of telomerase has been documented in over $90 \%$ of endometrial cancers [34,35]. Telomerase is comprised of an RNA template (hTR) and the catalytic protein hTERT which has reverse transcriptase activity. hTERT is considered to be the rate-limiting factor in the formation of functional telomerase.

Based on our previous work, the mTOR signaling pathway may also play a role in the regulation of telomerase activity. We have previously shown that rapamycin potently suppressed telomerase activity by decreasing hTERT mRNA levels in endometrial, ovarian and cervical cancer cell lines [12,24]. Although controversial, it has been suggested that hTERT transcription is cell cycle dependent and that non-cycling cells do not express hTERT $[36,37]$. Thus, one could argue that the effect of rapamycin on decreasing hTERT expression may occur simply as an indirect consequence of cell cycle arrest rather than a direct effect on hTERT transcription. However, we found that rapamycin globally decreased hTERT expression in gynecologic cancer cell lines, even in cell lines that were resistant to rapamycin's anti-proliferative effects and failed to undergo G1 arrest [24]. This is further supported by our findings in the type I and II endometrial cancer tissue explants. Rapamycin decreased hTERT expression in all of these tumors, independent of the responsiveness of these tumors to rapamycin's growth inhibitory effects. Thus, rapamycin may regulate hTERT mRNA expression through an alternative pathway downstream from mTOR that is distinct from its ability to induce cell cycle arrest.

Telomere length has also been implicated as a prognostic indicator in a number of solid tumors, including breast, colon, lung, prostate and hepatocellular carcinomas [38]. In general, alterations in telomere length are associated with worse clinical outcomes, but the type of alteration, i.e. short versus long telomeres, appears to be dependent on the tumor type [38]. Given the potential relationship between mTOR signaling and telomerase activity, we measured telomere length to see if this would correspond to response to treatment.

Although effects on hTERT expression failed to predict sensitivity to rapamycin among the endometrial cancer tissue explants, our hope was that differences in telomere length may be more helpful. However, the mean telomere length was the same between rapamycin sensitive and resistant tumors; and thus, was not a biomarker of response to this drug.

Biomarkers to predict sensitivity to mTOR inhibitors have remained elusive both in preclinical studies and in clinical trials for endometrial cancer as well as other tumor types. As 
demonstrated in our work, PTEN and phosphorylated Akt status failed to predict sensitivity to treatment with rapamycin among type I and type II endometrial cancer tumor explants. A number of other potential biomarkers were explored, including phosphorylated S6, phosphorylated 4E-BP-1, hTERT and telomere length, but none were found to be effective in discriminating which tumors would best respond to the anti-proliferative effects of rapamycin treatment. Our numbers are small, and this limitation could have impacted the emergence of one of these factors as a legitimate biomarker. However, these findings are consistent with those from recent clinical trials of mTOR inhibitors in endometrial cancer.

Three mTOR inhibitors have been or are currently being investigated in women with endometrial cancer: temsirolimus (CCI-779, Wyeth), everolimus (RAD001, Novartis) and ridaforolimus (formerly deforolimus, AP23573, Merck). According to the results from the completed phase II trials to date, mTOR inhibitors do exhibit activity in women with advanced or recurrent endometrial cancer [7], with response rates of 26-28\%. Based on the biologic correlates derived from some of these trials, response to mTOR inhibitors has not correlated with PTEN status or Akt and mTOR expression patterns [7]. Although loss of PTEN is more commonly associated with type I tumors, there is no indication that mTOR inhibitors are not equally efficacious in type II tumors and other molecular characteristics may ultimately be the more critical factor in predicting sensitivity or alternatively, resistance to treatment. It is possible that alternative approaches such as microarray gene expression profiling may lead to identification of biomarkers predicting sensitivity to mTOR inhibition. Thus, the search continues for meaningful biomarkers or combinations of biomarkers to help identify endometrial cancer patients who are most likely to benefit from mTOR inhibitor therapy, and this should continue to be a priority goal in subsequent clinical trials and translational studies.

\section{Acknowledgments}

This work was generously supported by the Lineberger Comprehensive Cancer Center Clinical/Translational Award, the V Foundation for Cancer Research, the UNC Clinical Translational Science Award-K12 Scholars Program (KL2 RR025746), and the Steelman Fund.

\section{References}

[1]. Jemal A, Siegel R, Ward E, Hao Y, Xu J, Thun MJ. Cancer statistics, 2009. CA Cancer J Clin. 2009; 59(4):225-49. [PubMed: 19474385]

[2]. Bokhman JV. Two pathogenetic types of endometrial carcinoma. Gynecol Oncol. 1983; 15(1):107. [PubMed: 6822361]

[3]. Abal M, Planaguma J, Gil-Moreno A, et al. Molecular pathology of endometrial carcinoma: transcriptional signature in endometrioid tumors. Histol Histopathol. 2006; 21(2):197-204. [PubMed: 16329044]

[4]. Hecht JL, Mutter GL. Molecular and pathologic aspects of endometrial carcinogenesis. J Clin Oncol. 2006; 24(29):4783-91. [PubMed: 17028294]

[5]. Lax SF. Molecular genetic pathways in various types of endometrial carcinoma: from a phenotypical to a molecular-based classification. Virchows Arch. 2004; 444(3):213-23. [PubMed: 14747944]

[6]. Ambros RA, Sherman ME, Zahn CM, Bitterman P, Kurman RJ. Endometrial intraepithelial carcinoma: a distinctive lesion specifically associated with tumors displaying serous differentiation. Hum Pathol. 1995; 26(11):1260-7. [PubMed: 7590702] 
[7]. Gehrig PA, Bae-Jump VL. Promising novel therapies for the treatment of endometrial cancer. Gynecol Oncol. 2010; 116(2):187-94. [PubMed: 19903572]

[8]. Mutter GL, Lin MC, Fitzgerald JT, et al. Altered PTEN expression as a diagnostic marker for the earliest endometrial precancers. J Natl Cancer Inst. 2000; 92(11):924-30. [PubMed: 10841828]

[9]. Cantley LC, Neel BG. New insights into tumor suppression: PTEN suppresses tumor formation by restraining the phosphoinositide 3-kinase/AKT pathway. Proc Natl Acad Sci U S A. 1999; 96(8): 4240-5. [PubMed: 10200246]

[10]. Pullen N, Thomas G. The modular phosphorylation and activation of p70s6k. FEBS Lett. 1997; 410(1):78-82. [PubMed: 9247127]

[11]. Sonenberg N, Gingras AC. The mRNA 5' cap-binding protein eIF4E and control of cell growth. Curr Opin Cell Biol. 1998; 10(2):268-75. [PubMed: 9561852]

[12]. Zhou C, Gehrig PA, Whang YE, Boggess JF. Rapamycin inhibits telomerase activity by decreasing the hTERT mRNA level in endometrial cancer cells. Mol Cancer Ther. 2003; 2(8): 789-95. [PubMed: 12939469]

[13]. Bieche I, Nogues C, Paradis V, et al. Quantitation of hTERT gene expression in sporadic breast tumors with a real-time reverse transcription-polymerase chain reaction assay. Clin Cancer Res. 2000; 6(2):452-9. [PubMed: 10690523]

[14]. Jefferies HB, Fumagalli S, Dennis PB, Reinhard C, Pearson RB, Thomas G. Rapamycin suppresses 5'TOP mRNA translation through inhibition of p70s6k. EMBO J. 1997; 16(12):3693704. [PubMed: 9218810]

[15]. Gingras AC, Raught B, Sonenberg N. Regulation of translation initiation by FRAP/mTOR. Genes Dev. 2001; 15(7):807-26. [PubMed: 11297505]

[16]. Pause A, Methot N, Svitkin Y, Merrick WC, Sonenberg N. Dominant negative mutants of mammalian translation initiation factor eIF-4A define a critical role for eIF-4F in cap-dependent and cap-independent initiation of translation. EMBO J. 1994; 13(5):1205-15. [PubMed: 8131750]

[17]. Bansal N, Yendluri V, Wenham RM. The molecular biology of endometrial cancers and the implications for pathogenesis, classification, and targeted therapies. Cancer Control. 2009; 16(1): 8-13. [PubMed: 19078924]

[18]. Oda K, Stokoe D, Taketani Y, McCormick F. High frequency of coexistent mutations of PIK3CA and PTEN genes in endometrial carcinoma. Cancer Res. 2005; 65(23):10669-73. [PubMed: 16322209]

[19]. Podsypanina K, Lee RT, Politis C, et al. An inhibitor of mTOR reduces neoplasia and normalizes p70/S6 kinase activity in Pten+/- mice. Proc Natl Acad Sci U S A. 2001; 98(18):10320-5. [PubMed: 11504907]

[20]. Shi Y, Gera J, Hu L, et al. Enhanced sensitivity of multiple myeloma cells containing PTEN mutations to CCI-779. Cancer Res. 2002; 62(17):5027-34. [PubMed: 12208757]

[21]. Grunwald V, DeGraffenried L, Russel D, Friedrichs WE, Ray RB, Hidalgo M. Inhibitors of mTOR reverse doxorubicin resistance conferred by PTEN status in prostate cancer cells. Cancer Res. 2002; 62(21):6141-5. [PubMed: 12414639]

[22]. Neshat MS, Mellinghoff IK, Tran C, et al. Enhanced sensitivity of PTEN-deficient tumors to inhibition of FRAP/mTOR. Proc Natl Acad Sci U S A. 2001; 98(18):10314-9. [PubMed: 11504908]

[23]. Noh WC, Mondesire WH, Peng J, et al. Determinants of rapamycin sensitivity in breast cancer cells. Clin Cancer Res. 2004; 10(3):1013-23. [PubMed: 14871980]

[24]. Bae-Jump VL, Zhou C, Gehrig PA, Whang YE, Boggess JF. Rapamycin inhibits hTERT telomerase mRNA expression, independent of cell cycle arrest. Gynecol Oncol. 2006; 100(3): 487-94. [PubMed: 16249016]

[25]. Yu K, Toral-Barza L, Discafani C, et al. mTOR, a novel target in breast cancer: the effect of CCI-779, an mTOR inhibitor, in preclinical models of breast cancer. Endocr Relat Cancer. 2001; 8(3):249-58. [PubMed: 11566616]

[26]. Yang L, Clarke MJ, Carlson BL, et al. PTEN loss does not predict for response to RAD001 (Everolimus) in a glioblastoma orthotopic xenograft test panel. Clin Cancer Res. 2008; 14(12): 3993-4001. [PubMed: 18559622] 
[27]. Breuleux M, Klopfenstein M, Stephan C, et al. Increased AKT S473 phosphorylation after mTORC1 inhibition is rictor dependent and does not predict tumor cell response to PI3K/mTOR inhibition. Mol Cancer Ther. 2009; 8(4):742-53. [PubMed: 19372546]

[28]. Boulay A, Zumstein-Mecker S, Stephan C, et al. Antitumor efficacy of intermittent treatment schedules with the rapamycin derivative RAD001 correlates with prolonged inactivation of ribosomal protein S6 kinase 1 in peripheral blood mononuclear cells. Cancer Res. 2004; 64(1): 252-61. [PubMed: 14729632]

[29]. Peralba JM, DeGraffenried L, Friedrichs W, et al. Pharmacodynamic evaluation of CCI-779, an inhibitor of mTOR, in cancer patients. Clin Cancer Res. 2003; 9(8):2887-92. [PubMed: 12912932]

[30]. Tabernero J, Rojo F, Calvo E, et al. Dose- and schedule-dependent inhibition of the mammalian target of rapamycin pathway with everolimus: a phase I tumor pharmacodynamic study in patients with advanced solid tumors. J Clin Oncol. 2008; 26(10):1603-10. [PubMed: 18332469]

[31]. Dudkin L, Dilling MB, Cheshire PJ, et al. Biochemical correlates of mTOR inhibition by the rapamycin ester CCI-779 and tumor growth inhibition. Clin Cancer Res. 2001; 7(6):1758-64. [PubMed: 11410517]

[32]. Houghton PJ. Everolimus. Clin Cancer Res. 2010; 16(5):1368-72. [PubMed: 20179227]

[33]. Stewart SA, Weinberg RA. Telomerase and human tumorigenesis. Semin Cancer Biol. 2000; 10(6):399-406. [PubMed: 11170862]

[34]. Yokoyama Y, Takahashi Y, Shinohara A, Lian Z, Tamaya T. Telomerase activity in the female reproductive tract and neoplasms. Gynecol Oncol. 1998; 68(2):145-9. [PubMed: 9514809]

[35]. Zheng PS, Iwasaka T, Yamasaki F, et al. Telomerase activity in gynecologic tumors. Gynecol Oncol. 1997; 64(1):171-5. [PubMed: 8995569]

[36]. Zhu X, Kumar R, Mandal M, et al. Cell cycle-dependent modulation of telomerase activity in tumor cells. Proc Natl Acad Sci U S A. 1996; 93(12):6091-5. [PubMed: 8650224]

[37]. Holt SE, Aisner DL, Shay JW, Wright WE. Lack of cell cycle regulation of telomerase activity in human cells. Proc Natl Acad Sci U S A. 1997; 94(20):10687-92. [PubMed: 9380696]

[38]. Svenson U, Roos G. Telomere length as a biological marker in malignancy. Biochim Biophys Acta. 2009; 1792(4):317-23. [PubMed: 19419696] 


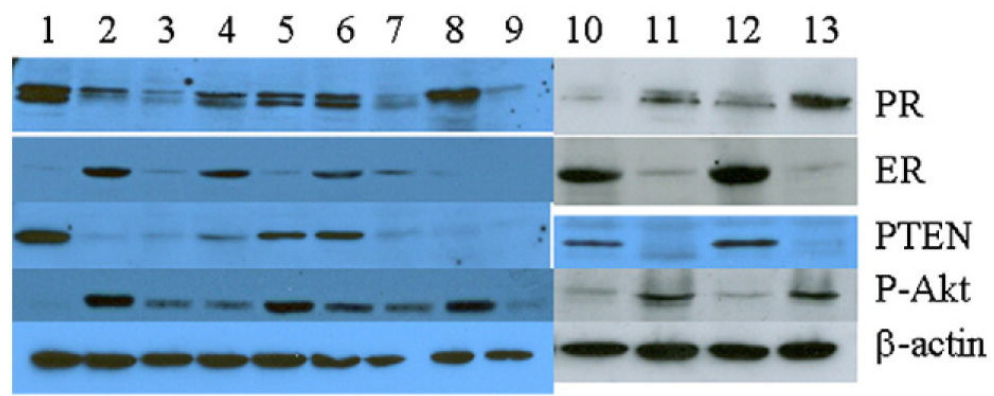

Fig. 1.

Expression of the progesterone receptor (PR), estrogen receptor (ER), PTEN, phosphorylated-AKT (P-Akt) and $\beta$-actin in 13 human endometrial cancer tumor explants by Western blotting. 

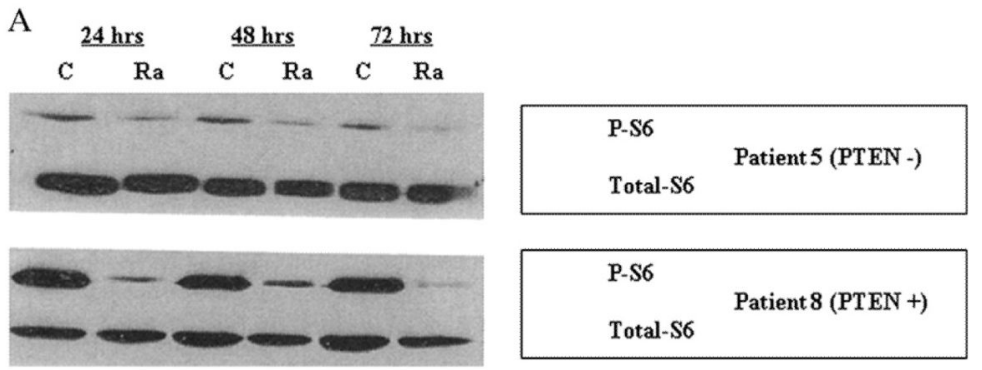

B

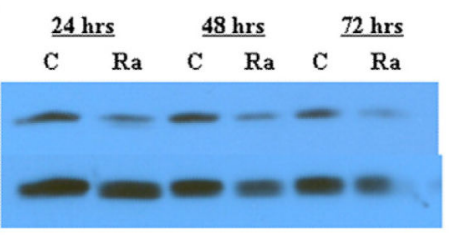

P-4E-BP-1

Total-4E-BP-1

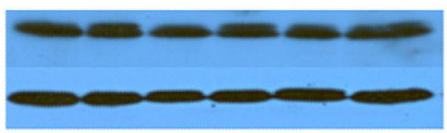

$$
\begin{aligned}
& \text { P-4E-BP-1 } \\
& \text { Total-4E-BP-1 }
\end{aligned}
$$

Fig. 2.

Rapamycin inhibited phosphorylation of S6 in all of the human endometrial cancer tumor explants as determined by Western blotting [A]. In addition, rapamycin decreased phosphorylation of 4E-BP-1 in most of these tumor explants (77\% or 10/13) [B]. Primary tumor cells were cultured for $24 \mathrm{~h}$ and then treated with $20 \mathrm{nM}$ rapamycin (Ra) or control (C) for 24,48 or $72 \mathrm{~h}$. Little effect was seen on total S6 or total 4E-BP-1. This is a representative Western blot of 4 of the 13 endometrial cancer tumor explants examined. 


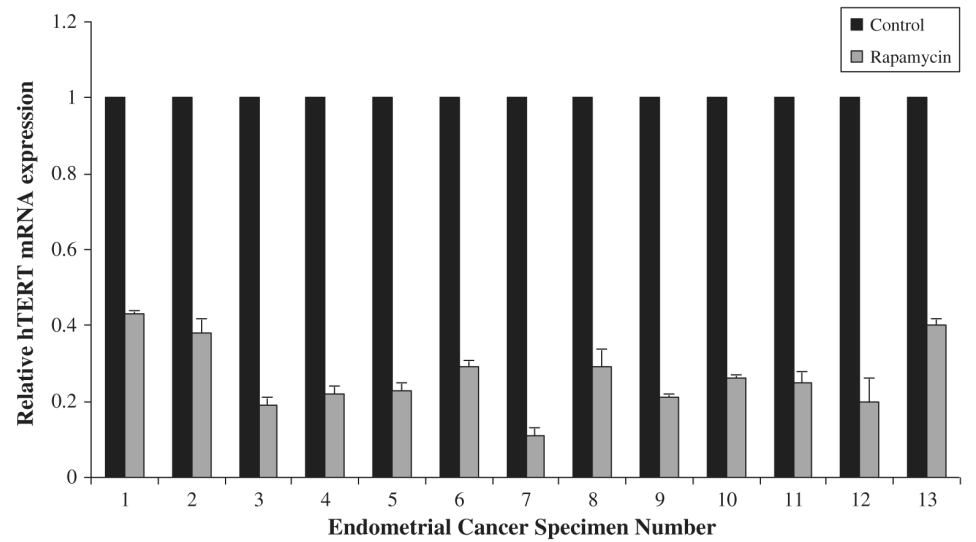

Fig. 3.

Rapamycin inhibits hTERT mRNA expression in human endometrial cancer tumor explants. Primary tumor cells were cultured for $48 \mathrm{~h}$ and then treated with $20 \mathrm{nM}$ rapamycin or control for $48 \mathrm{~h}$. hTERT mRNA levels were determined by real-time RT-PCR. 


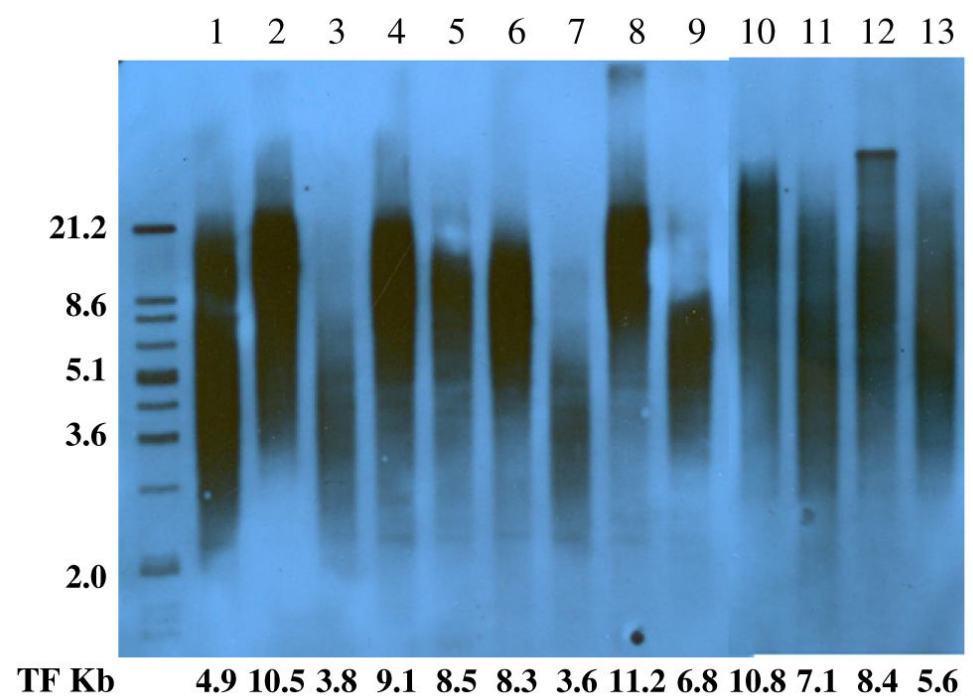

Fig. 4.

Comparison of telomere length in human endometrial cancer tumor explants by terminal restriction fragment Southern blot analysis. Telomere length did not predict responsiveness to rapamycin among these samples. 
Table 1

Characterization of endometrial cancer explants treated with rapamycin.

\begin{tabular}{lllllll}
\hline Patient & Histology & Grade & $\begin{array}{l}\text { FIGO } \\
\text { stage }\end{array}$ & $\begin{array}{l}\text { IC }_{50} \\
(\mathbf{n M})\end{array}$ & Wildtype-PTEN & Phospho-Akt \\
\hline 1 & endometrioid & 2 & IB & $\mathrm{a}$ & + & - \\
2 & endometrioid & 3 & IB & 50 & - & + \\
3 & serous & 3 & IA & 0.01 & - & + \\
4 & endometrioid & 3 & IB & 1 & + & + \\
5 & endometrioid & 3 & IB & 10 & + & + \\
6 & clear & 3 & IB & $\mathrm{a}$ & + & + \\
7 & endometrioid & 1 & IB & 1 & + & + \\
8 & serous & 3 & IB & $\mathrm{a}$ & - & + \\
9 & endometrioid & 3 & IB & $\mathrm{a}$ & - & + \\
10 & clear & 3 & IVB & 15 & + & + \\
11 & endometrioid & 1 & IB & 0.01 & - & + \\
12 & endometrioid & 2 & IA & 25 & + & + \\
13 & endometrioid & 1 & IA & 0.5 & - & + \\
\hline
\end{tabular}

${ }^{a}$ Did not respond to treatment with rapamycin.

\footnotetext{
This work was presented in part at the 2005 Annual Meeting of the Society of Gynecologic Oncologists, Miami Beach, FL. This work was presented in part at the 2005 Annual Meeting of the Society of Gynecologic Oncologists, Miami Beach, FL. ${ }^{2}$ This work was presented in part at the 2005 Annual Meeting of the Society of Gynecologic Oncologists, Miami Beach, FL. ${ }^{2}$ This work was presented in part at the 2005 Annual Meeting of the Society of Gynecologic Oncologists, Miami Beach, FL.
} 


\section{Table 2}

Rapamycin in type I and type II endometrial cancers.

\begin{tabular}{lll}
\hline Type & Type I $(\boldsymbol{n}=\mathbf{9})$ & Type II $(\boldsymbol{n}=\mathbf{4})$ \\
\hline Mean $\mathrm{IC}_{50}$ & $\begin{array}{l}12.5 \mathrm{nM}(7 / 9) \text { samples } \\
\text { responded }\end{array}$ & $\begin{array}{l}7.5 \mathrm{nM}(2 / 4 \text { samples } \\
\text { responded })\end{array}$ \\
ER status & $6 / 9$ positive & $3 / 4$ positive \\
PR status & $9 / 9$ positive & $4 / 4$ positive \\
Wildtype-PTEN status & $5 / 9$ positive & $2 / 4$ positive \\
Phospho-Akt status & $8 / 9$ positive & $4 / 4$ positive \\
Phospho-S6 & $\begin{array}{l}\text { Decreased by } \\
\text { rapamycin in } 9 / 9\end{array}$ & $\begin{array}{l}\text { Decreased by } \\
\text { rapamycin in } 4 / 4\end{array}$ \\
Phospho-4E-BP-1 & $\begin{array}{l}\text { Decreased by } \\
\text { rapamycin in } 7 / 9\end{array}$ & $\begin{array}{l}\text { Decreased by } \\
\text { rapamycin in } 4 / 4\end{array}$ \\
hTERT mRNA expression & $\begin{array}{l}\text { Decreased by } \\
\text { rapamycin in } 9 / 9\end{array}$ & $\begin{array}{l}\text { Decreased by } \\
\text { rapamycin in } 4 / 4\end{array}$ \\
Mean telomere length & $7.2 \mathrm{~kb}$ & $8.5 \mathrm{~kb}$ \\
\hline
\end{tabular}

